\title{
Local analytic sector subtraction for NNLO QCD calculations
}

\section{Giovanni Pelliccioli*}

Dipartimento di Fisica and Arnold-Regge Center, Università di Torino, and INFN, Sezione di Torino, Via P. Giuria 1, I-10125 Torino, Italy

E-mail: gpellicc@to.infn.it

We present a new local, analytic scheme for the subtraction of infrared singularities at next-tonext-to-leading order (NNLO) in QCD, which aims at reducing the complexity of the problem and features remarkable aspects. It works for any infrared-safe observable, it benefits from the partition of the radiative phase-space into sectors, the subtraction counterterms are local and can be analytically integrated. All these properties enable an efficient numerical implementation. Our scheme is currently designed for massless final state radiation only, but its extension to initial state radiation will allow for predictions at hadron-hadron and lepton-nucleon colliders.

XXVII International Workshop on Deep-Inelastic Scattering and Related Subjects - DIS2019

8-12 April, 2019

Torino, Italy

${ }^{*}$ Speaker. 


\section{Introduction}

The computation of NNLO QCD corrections is becoming mandatory to provide accurate fixedorder predictions for high-energy colliders processes. Beyond leading order (LO), virtual and real radiation terms contribute. Even if such terms separately generate infrared (IR) singularities, their combination (assuming UV renormalization) gives finite predictions for physical observables. However, the complexity of amplitudes structures requires a method to evaluate numerically the contributions after getting rid of IR divergences, and the subtraction procedure achieves this goal.

At next-to-leading order (NLO) the main subtraction schemes are Frixione-Kunszt-Signer (FKS) [1] and Catani-Seymour (CS) [2]. At NNLO, a large number of subtraction procedures have been developed and used to obtain phenomenological results at high-energy colliders results $[3,4,5,6,7,8]$. New ideas are being developed $[9,10,11]$ and slicing methods are already available $[12,13,14]$. However, the proposed NNLO schemes are characterized by an increased complexity if compared with the NLO ones, and many of them do not feature desirable aspects such as analyticity and locality of the counterterms.

In this contribution, we present a new scheme [15] which aims at reducing the complexity of the subtraction problem at NNLO. The proposed scheme is designed for massless final state radiation, thus in all the following we consider processes with partons in the final state only.

\section{NLO}

In order to build a new subtraction procedure with minimal structure, we have first analyzed the most advantageous aspects which have been successfully employed in pre-existing NLO schemes, with a view to exporting them to NNLO.

Let's consider a process which features $n$ final state partons at LO. Given an IR-safe observable $X$, the NLO correction to the differential cross-section in $X$ receives contribution from a one-loop virtual term, $V$, evaluated with $n$-body kinematics, and from a single-real radiation term, $R$, evaluated with $n+1$-body kinematics. In $d=4-2 \varepsilon$ dimensions, the explicit $\varepsilon$ poles of virtual orgin are exactly cancelled by those arising from the integration of $R$ over the additional radiation phasespace (KLN theorem). Then the complete NLO cross-section is finite in $d=4$. However, due to the complexity of the virtual and real terms structure (which must be integrated in $d=4-2 \varepsilon$ dimensions), and due to the complexity of the $X$-observable, the calculation is in general very complicated. The subtraction procedure allows to perform the entire computation in four dimensions. It consists in adding and subtracting a local counterterm $K_{n+1}$, which features the same IR singularities as $R$ in the one-unresolved regime, but is much easier than $R$ to be integrated analitically over the unresolved parton phase-space. Relying on phase-space factorization and definining $I_{n}$ as the integral of $K_{n+1}$ over the radiative phase-space, we obtain the subtracted NLO correction to the $X$ distribution, which is

$$
\frac{d \sigma_{\mathrm{NLO}}}{d X}=\int d \Phi_{n}\left(V+I_{n}\right) \delta_{n}(X)+\int d \Phi_{n+1}\left(R \delta_{n+1}(X)-K_{n+1} \delta_{n}(X)\right),
$$

where the first and the second integrand are separately finite and integrable in $d=4$. The longknown FKS and CS subtraction schemes which are widely used in the literature have proved very successfull at NLO, but they feature several bootlenecks when trying to extend them to NNLO. 
Taking inspiration from the advantages of both methods, we have built a new NLO scheme which features a minimal singularity structure and the partition of radiative phase-space into sectors, as in FKS. At the same time, in a similar fashion as in CS, the proposed counterterms are sum of terms, each parameterized with a different kinematic mapping, and allow for simplified analytic integration. All of these aspects can be exported naturally to NNLO.

\section{NNLO}

The NNLO correction to the differential cross-section in the observable $X$ receives contributions from a double-real term $R R$, and from UV renormalised double-virtual $V V$ and real-virtual $R V$ terms. The subtraction procedure consists in adding and subtracting counterterms reproducing the singularities of $R R$ and $R V$. We introduce $\int d \Phi_{n+2}\left[K^{(\mathbf{1})} \delta_{n+1}+\left(K^{(\mathbf{1 2})}+K^{(\mathbf{2})}\right) \delta_{n}\right]$, where $K^{(\mathbf{1})}$ and $\left(K^{(\mathbf{1 2})}+K^{(\mathbf{2})}\right)$ feature the same one- and two-unresolved limits of $R R$, respectively. Similarly, we introduce $\int d \Phi_{n+1} K^{(\mathbf{R V})} \delta_{n}$, where $K^{(\mathbf{R V})}$ has the same phase-space divergences of $R V$.

Calling $I^{(\mathbf{1})}, I^{(\mathbf{2})}, I^{(\mathbf{1 2})}$ and $I^{(\mathbf{R V})}$ the correspondent integrated counterterms, the subtracted cross section reads

$$
\begin{aligned}
d \sigma_{\mathrm{NNLO}} / d X & =\int d \Phi_{n}\left(V V+I^{(\mathbf{2})}+I^{(\mathbf{R V})}\right) \delta_{n} \\
& +\int d \Phi_{n+1}\left[\left(R V+I^{(\mathbf{1})}\right) \delta_{n+1}-\left(K^{(\mathbf{R V})}-I^{(\mathbf{1 2})}\right) \delta_{n}\right] \\
& +\int d \Phi_{n+2}\left[R R \delta_{n+2}-K^{(\mathbf{1})} \delta_{n+1}-\left(K^{(\mathbf{1 2})}+K^{(\mathbf{2})}\right) \delta_{n}\right]
\end{aligned}
$$

$I^{(\mathbf{1})}$ has the same $1 / \varepsilon$ poles as $R V, I^{(\mathbf{1 2})}$ has the same $1 / \varepsilon$ poles as $K^{(\mathbf{R V})}$, while the sum $I^{(\mathbf{2})}+I^{(\mathbf{R V})}$ has the same $1 / \varepsilon$ poles as $V V$. The terms $\left(R V+I^{(\mathbf{1})}\right)$ and $\left(K^{(\mathbf{R V})}-I^{(\mathbf{1 2})}\right)$ still feature phase-space singularities in $\Phi_{n+1}$, but their difference does not. This ensures that each of the three lines is finite in $d=4$ and suitable for numerical integration.

In addition to the one-unresolved soft $\mathbf{S}_{i}$ (soft parton $i$ ) and collinear $\mathbf{C}_{i j}$ (collinear partons $i, j$ ) configurations that already appear in NLO calculations, at NNLO two-unresolved configurations arise: the double-soft $\mathbf{S}_{i j}$ (uniformly soft partons $i, j$ ), the double-collinear $\mathbf{C}_{i j k}$ (partons $i, j, k$ uniformly collinear) and $\mathbf{C}_{i j k l}\left(i, j\right.$ and $k, l$ separatey collinear), and the soft-collinear $\mathbf{S C}_{i j k}$ (soft parton $i$ and collinear partons $j, k$ ). The one- and two-unresolved limits of $R R$ feature a general structure $[16,17]$ characterized by universal singular kernels multiplyed by Born-level matrix-elements.

In a similar fashion as at NLO, we partition the double-radiative phase-space by means of sector functions $\mathscr{W}_{i j k l},\left(\sum_{i j k l} \mathscr{W}_{i j k l}=1\right)$, such that each sector embeds a minimum number of singular configurations. Sector functions are defined in terms of Lorentz invariants $s_{i j}=2 p_{i} \cdot p_{j}$ (indices run over the $n+2$ final partons), and they obey sum rules which are crucial for the analytic integration of counterterms. In fact, summing over sectors sharing the same singularity, and taking the correspondent singular limit on the sum, $\mathscr{W}$ functions disappear. Another noticeable feature of sector functions is that when considering single-unresolved configurations, NNLO sector functions factorise NLO sector functions, allowing $\left(R V+I^{(\mathbf{1})}\right)$ and $\left(K^{(\mathbf{R V})}-I^{(\mathbf{1 2})}\right)$ to be finite in $d=4$ NLO sector by NLO sector.

In each sector $\mathscr{W}_{a b c d}$ we define a candidate local counterterm which catches the IR singular structure of $R R$ in that sector. Let's take a sector of the type $\mathscr{W}_{i j k j}$, which contains the following 
singular limits: $\mathbf{S}_{i}, \mathbf{C}_{i j}, \mathbf{S}_{i k}, \mathbf{C}_{i j k}, \mathbf{S C}_{i j k}, \mathbf{C S}_{i j k}$. Note that it is crucial that $\mathbf{S C}_{i j k}, \mathbf{C S}_{i j k}$ are opposite hierarchic soft-collinear limits: $\mathbf{S C}_{i j k}$ features a parton $i$ going soft more quickly than $j, k$ going collinear, while $\mathbf{C S}_{i j k}$ features two partons $i, j$ approaching the collinear regime more quickly than $i$ going soft. The candidate counteterms are:

$$
\begin{aligned}
K_{i j k j}^{(\mathbf{1})} & =\left[\mathbf{S}_{i}+\mathbf{C}_{i j}\left(1-\mathbf{S}_{i}\right)\right] R R \mathscr{W}_{i j k j}, \\
K_{i j k j}^{(\mathbf{2})} & =\left[\mathbf{S}_{i k}+\mathbf{C}_{i j k}\left(1-\mathbf{S}_{i k}\right)+\left(\mathbf{S C}_{i j k}+\mathbf{C S}_{i j k}\right)\left(1-\mathbf{S}_{i k}\right)\left(1-\mathbf{C}_{i j k}\right)\right] R R \mathscr{W}_{i j k j}, \\
K_{i j k j}^{(\mathbf{1 2})} & =-\left[\mathbf{S}_{i}+\mathbf{C}_{i j}\left(1-\mathbf{S}_{i}\right)\right]\left[\mathbf{S}_{i k}+\mathbf{C}_{i j k}\left(1-\mathbf{S}_{i k}\right)+\left(\mathbf{S C}_{i j k}+\mathbf{C S}_{i j k}\right)\left(1-\mathbf{S}_{i k}\right)\left(1-\mathbf{C}_{i j k}\right)\right] R R \mathscr{W}_{i j k j},
\end{aligned}
$$

Similar expressions can be written for other sector topologies $\left(\mathscr{W}_{i j j k}, \mathscr{W}_{i j k l}\right)$. The treatment of the real-virtual counterterm $R V$ will be detailed in a future publication.

Simplifications are possible in the structure of counterterms shown in Eq. 3.2, such as the cancellation of soft-collinear terms $\mathbf{S C}_{i j k}, \mathbf{C S}_{i j k}$ in the sum $K^{(\mathbf{2})}+K^{(\mathbf{1 2})}$. We stress that for our scheme it is crucial that singular limits commute when applied to sector functions or to matrix-elements.

In order to allow for analytic integration of the counterterms, we need to factorize the radiative phase-space from the Born phase-space. To do so, we choose a specific parameterization for each term of the counterterms, employing CS mappings which adapt to the Lorentz invariants appearing in the singular kernels. Thanks to the factorization properties and the sum rules of sector functions, we are then left with simplified counterterms which can be integrated analytically in $d$ dimensions. For more details we refer to Ref. [15].

In order to validate our subtraction scheme, we have considered the NNLO corrections to $e^{+} e^{-} \rightarrow j j$ which are proportional to $T_{R} C_{F}$. We have compared the inclusive cross-section obtained with the subtraction procedure with the analytic result, finding very good agreement. Even the renormalization scale dependence is correctly described, as shown in Fig. 1.

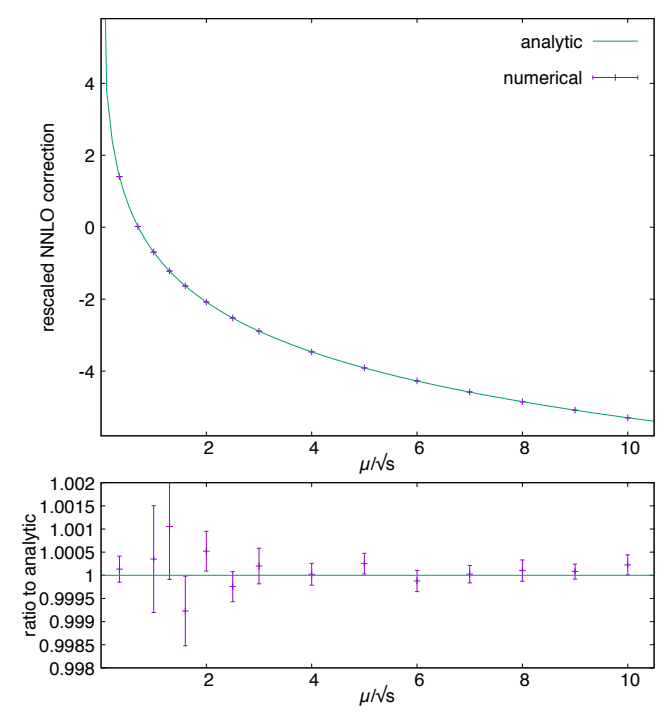

Figure 1: Rescaled NNLO correction as a function of the renormalisation scale. 


\section{Conclusions}

We have proposed a new NNLO subtraction scheme which features local counterterms that can be integrated analytically, is valid for any IR-safe observable, benefits from the partition of the radiative phase-space into sectors, and is numerically efficient. The presented scheme is currently applicable for processes with (massless) partons in the final state only, but its generalization to processes with hadrons in the initial state is expected in the early future.

\section{References}

[1] S. Frixione, Z. Kunszt and A. Signer, Nucl. Phys. B 467 (1996) 399 [hep-ph/9512328]. S. Frixione, Nucl. Phys. B 507 (1997) 295 [hep-ph/9706545].

[2] S. Catani and M. H. Seymour, Nucl. Phys. B 485 (1997) 291 [hep-ph/9605323]. S. Catani, S. Dittmaier, M. H. Seymour and Z. Trocsanyi, Nucl. Phys. B 627 (2002) 189 [hep-ph/0201036].

[3] A. Gehrmann-De Ridder, T. Gehrmann and E. W. N. Glover, JHEP 0509 (2005) 056 [hep-ph/0505111]. A. Daleo, T. Gehrmann and D. Maitre, JHEP 0704 (2007) 016 [hep-ph/0612257].

[4] M. Czakon, Phys. Lett. B 693 (2010) 259 [arXiv:1005.0274 [hep-ph]]. M. Czakon, Nucl. Phys. B 849 (2011) 250 [arXiv:1101.0642 [hep-ph]].

[5] R. Boughezal, K. Melnikov and F. Petriello, Phys. Rev. D 85 (2012) 034025 [arXiv:1111.7041 [hep-ph]].

[6] G. Somogyi, Z. Trocsanyi and V. Del Duca, JHEP 0506 (2005) 024 [hep-ph/0502226]. G. Somogyi, Z. Trocsanyi and V. Del Duca, JHEP 0701 (2007) 070 [hep-ph/0609042].

[7] S. Frixione and M. Grazzini, JHEP 0506 (2005) 010 [hep-ph/0411399].

[8] M. Cacciari, F. A. Dreyer, A. Karlberg, G. P. Salam and G. Zanderighi, Phys. Rev. Lett. 115 (2015) no.8, 082002 [arXiv:1506.02660 [hep-ph]].

[9] G. F. R. Sborlini, F. Driencourt-Mangin and G. Rodrigo, JHEP 1610 (2016) 162 doi:10.1007/JHEP10(2016)162 [arXiv:1608.01584 [hep-ph]].

[10] F. Caola, K. Melnikov and R. RÃüntsch, Eur. Phys. J. C 77 (2017) no.4, 248 [arXiv:1702.01352 [hep-ph]].

[11] F. Herzog, JHEP 1808 (2018) 006 doi:10.1007/JHEP08(2018)006 [arXiv:1804.07949 [hep-ph]].

[12] S. Catani and M. Grazzini, Phys. Rev. Lett. 98 (2007) 222002 [hep-ph/0703012].

[13] R. Boughezal, C. Focke, X. Liu and F. Petriello, Phys. Rev. Lett. 115 (2015) no.6, 062002 [arXiv:1504.02131 [hep-ph]].

[14] J. Gaunt, M. Stahlhofen, F. J. Tackmann and J. R. Walsh, JHEP 1509 (2015) 058 [arXiv:1505.04794 [hep-ph]].

[15] L. Magnea, E. Maina, G. Pelliccioli, C. Signorile-Signorile, P. Torrielli and S. Uccirati, JHEP 1812 (2018) 107 Erratum: [JHEP 1906 (2019) 013] doi:10.1007/JHEP06(2019)013, 10.1007/JHEP12(2018)107 [arXiv:1806.09570 [hep-ph]].

[16] S. Catani and M. Grazzini, Nucl. Phys. B 570 (2000) 287 [hep-ph/9908523].

[17] F. A. Berends and W. T. Giele, Nucl. Phys. B 313 (1989) 595. 